Dear author,

Please note that changes made in the online proofing system will be added to the article before publication but are not reflected in this PDF.

We also ask that this file not be used for submitting corrections. 


\title{
Q1 Dynamic correspondence principle in the viscoelasticity of metallic glasses
}

\author{
Q3 Q2 Guo-Jian Lyu ${ }^{\text {a,b }}$, Ji-Chao Qiao a,b ${ }^{\text {a, }}$ Yao Yao ${ }^{\text {a,c,* }}$, Jean-Marc Pelletier ${ }^{\text {b }}$, David Rodney ${ }^{\text {d, }}$ \\ Julien Morthomas ${ }^{\mathrm{b}}$, Claudio Fusco ${ }^{\mathrm{b}, * *}$ \\ a School of Mechanics, Civil Engineering Architecture, Northwestern Polytechnical University, Xi'an 710072, PR China \\ b Université de Lyon, MATEIS, UMR CNRS 5510, INSA-Lyon, F-69621 Villeurbanne cedex, France \\ c Max Planck Inst Eisenforsch GmbH, Max Planck Str 1, D-40237 Dusseldorf, Germany \\ d Institut Lumière Matière, UMR5306 Université Lyon 1-CNRS, Université de Lyon, F-69622 Villeurbanne Cedex, France
}

\section{A R T I C L E I N F O}

Article history:

Received 2 July 2019

Accepted 11 August 2019

Available online $\mathrm{xxxx}$

\section{Keywords:}

Metallic glass

Dynamic mechanical relaxations

Molecular dynamics simulations

Dynamic correspondence principle

Viscoelasticity

Dynamic Poisson's ratio

\begin{abstract}
A B S T R A C T
We simulate dynamical mechanical spectroscopy in a $\mathrm{Cu}_{64} \mathrm{Zr}_{36}$ bulk metallic glass using non-equilibrium molec- 20 ular dynamics. Applying several loading conditions (constant volume, longitudinal, uniaxial and isostatic), we 21 find that different elastic moduli have very contrasted dynamical properties but satisfy the dynamic correspon- 22 dence principle, which states that the relations between static moduli can be extended to dynamical moduli, both 23 below and above the glass transition temperature. In particular, we determine the debated dynamic Poisson's 24 ratio from three different but consistent expressions. Finally, we trace the origin of dissipation down to regions 25 of low stability devoid of icosahedral clusters. (C) 2019 Acta Materialia Inc. Published by Elsevier Ltd. All rights reserved. 27
Dynamic mechanical spectroscopy (DMS) is a widespread method to measure complex dynamic elastic moduli and characterize the viscoelastic properties of materials [1]. In the case of metallic glasses (MGs) of particular interest here [2], DMS has been used to evaluate internal friction, i.e. the energy dissipated during cyclic deformation, a key engineering parameter for a number of applications such as resonating micro-electromechanical systems (MEMS) [3]. On a more fundamental level, DMS has also been used to study the intricate relaxation dynamics characteristic of disordered solids [4,5] and in particular the primary $(\alpha)$ and secondary $(\beta)$ relaxations $[4,6]$.

Experimentally, two modes of deformation are applied, bending [7-9] and torsion [10-12], giving access respectively to the complex Young and shear moduli. DMS has also been simulated using nonequilibrium molecular dynamics (NEMD) [13-17]. The simulations are limited to rather high frequencies $(>1 \mathrm{GHz}$ ) but allow to simultaneously measure the dissipation and analyze its origin at the atomic level. In particular, it has been shown that in metallic glasses, atoms with stable icosahedral environments do not participate in dissipation [13].

In the static limit, there are only two independent elastic moduli in isotropic media [18]. They come as pairs, such as $(\lambda, G)$, with $\lambda$ Lamé's first parameter and $G$ the shear modulus, and $(E, \nu)$, with $E$ Young's modulus and $\nu$ Poisson's ratio. These moduli are related to one another,

\footnotetext{
* Correspondence to: Y. Yao, School of Mechanics, Civil Engineering Architecture Northwestern Polytechnical University, Xi'an 710072, PR China.

** Corresponding author.

E-mail addresses: yaoy@nwpu.edu.cn (Y. Yao), claudio.fusco@insa-lyon.fr (C. Fusco).
}

with for instance, $G=E / 2(1+\nu)$. Therefore, if one can measure any 61 two moduli, all other moduli are known. These relations are well 62 established in static elasticity and derive from Hooke's law. It has been 63 assumed that they also hold true in the dynamic case, through the so- 64 called dynamic correspondence principle $[19,20]$, which simply means 65 that real static moduli can be replaced by their complex dynamic coun- 66 terparts. However, concerns have been raised about the dynamic 67 Poisson's ratio [21-24]. Also, the correspondence principle has actually 68 never been tested experimentally because it requires a precision on 69 the complex moduli very difficult to reach [21]. It has also not been 70 tested in NEMD, which to the best of our knowledge, has so far only 71 been applied with shear deformations. At high frequencies, in the har- 72 monic regime, the dynamic elastic moduli can be expressed analytically 73 [25] and satisfy the correspondence principle. However, the same is far 74 from clear in the anharmonic regime observed in MGs.

Here, we consider a typical CuZr MG and use NEMD to apply differ- 76 ent loading geometries and test the dynamic correspondence principle 77 over a wide range of frequencies and temperatures. We find that differ- 78 ent elastic moduli exhibit very different dynamic properties but that the 79 correspondence principle applies both below and above the glass tran- 80 sition temperature. We also devote a special discussion to the compli- 81 cated case of Poisson's ratio.

The MD simulations were performed using the open source 83 LAMMPS package [26]. We considered a $\mathrm{Cu}_{64} \mathrm{Zr}_{36} \mathrm{MG}$, which has a 84 high glass forming ability and has been widely investigated by MD sim- 85 ulations in the past [27-32]. The interatomic interactions are based on 86 the EAM potential developed by Mendelev et al. [33]. We simulated a 87 
glass with 108,000 atoms (see Fig. 1(a)), obtained by quenching a liquid from $2000 \mathrm{~K}$ down to $100 \mathrm{~K}$ with a typical quenching rate of $10^{11} \mathrm{~K} / \mathrm{s}$, keeping the pressure to zero during the cooling procedure.

We simulated DMS using the same methodology as in previous NEMD works $[13,16,25]$. Fig. 1 illustrates the case of pure shear. The sample was first equilibrated at the target temperature and zero pressure for $1 \mathrm{~ns}$. As shown in Fig. 1(a), we then applied a sinusoidal strain $\varepsilon_{x x}(t)=\varepsilon_{A} \sin (\omega t)$ along the X direction, with $\varepsilon_{A}$ and $T_{\omega}=2 \pi / \omega$, the amplitude and period of the cyclic deformation. In order to impose a pure shear and keep the volume of the cell constant, we applied strains $\varepsilon_{y y}(t)=\varepsilon_{z z}(t)=-0.5 \varepsilon_{x x}(t)$ along the $\mathrm{Y}$ and $\mathrm{Z}$ directions. We tested different strain amplitudes (see
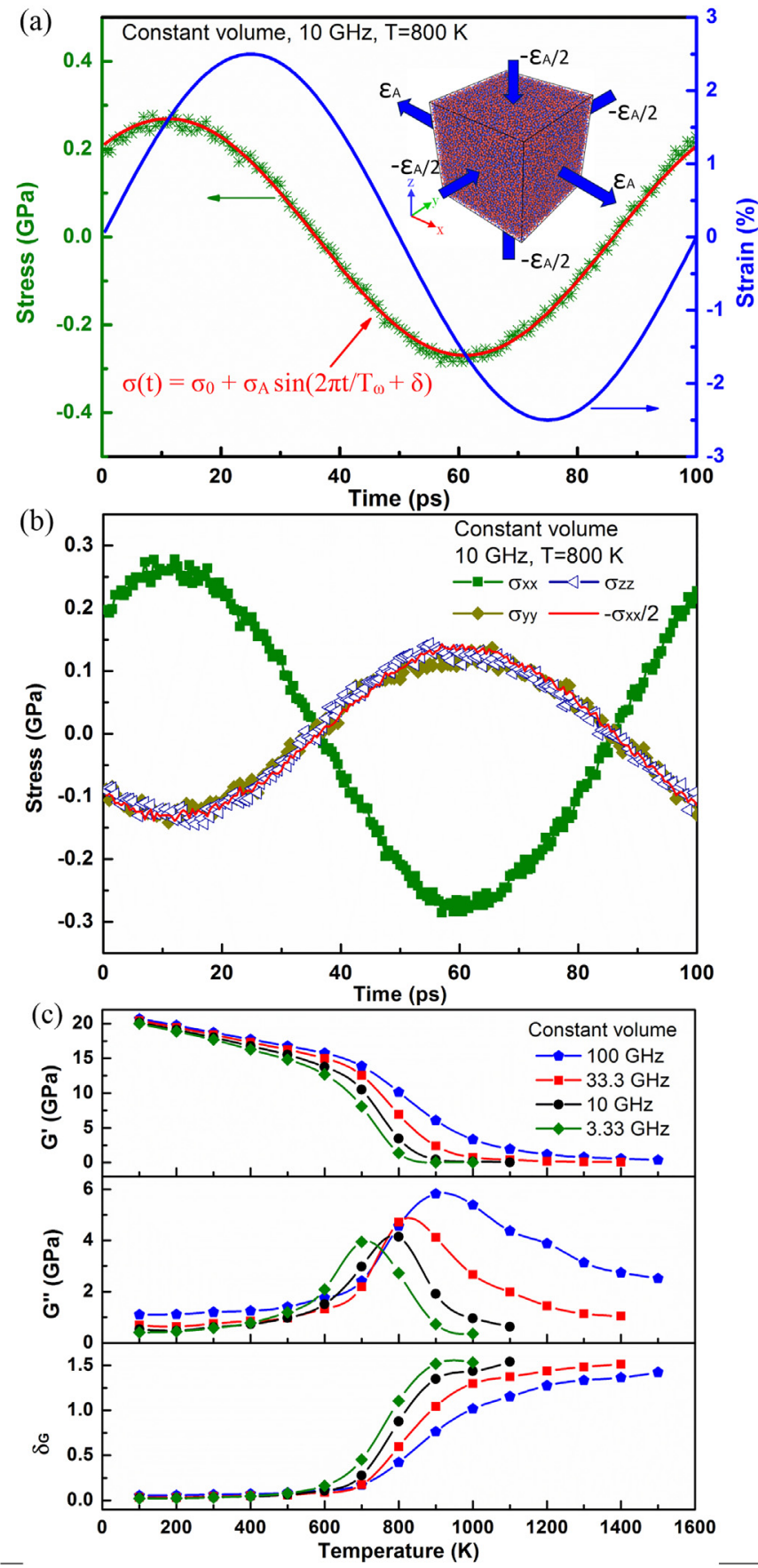

Fig. 1. Viscoelasticity in pure shear: (a) Time-dependent applied strain and resulting stress in $\mathrm{X}$ direction at $800 \mathrm{~K}$ and a frequency of $10 \mathrm{GHz}\left(T_{\omega}=100 \mathrm{ps}\right)$. The inset shows the atomic configuration and a schematic of the loading. (b) Tensile stresses in all three directions. (c) Storage shear modulus $G^{\prime}$, loss modulus $G^{\prime \prime}$ and phase shift $\delta_{G}$ as a function of temperature for different loading frequencies noted in the figure.
Supplementary materials) and found $\varepsilon_{A}=2.5 \%$ an optimal value, small 99 enough to remain in the elastic regime but large enough to avoid a poor 100 signal-to-noise ratio at high temperatures. We also simulated different 101 numbers of cycles (see Supplementary materials), and found that 102 5 cycles are enough to measure accurately dynamic elastic moduli. The 103 variation of the stress tensor was recorded during the deformation. As il- 104 lustrated in Fig. 1(a), after shifting all values by periodicity in the first pe- 105 riod, we fitted the stress in the X direction as $\sigma_{x x}(t)=\sigma_{0}+\sigma_{A} \sin (\omega t+106$ $\delta$ ), with $\sigma_{A}$ the stress amplitude and $\delta$ the phase shift. Discarding the small 107 offset stress $\sigma_{0}$, we have in complex notation, $\sigma_{x x}(t)=\sigma_{A} / \varepsilon_{A} \exp (i \delta) \varepsilon_{x x}(t) .108$ According to Hooke's law:

$\boldsymbol{\sigma}=2 G \boldsymbol{\varepsilon}+\lambda \operatorname{Tr}(\boldsymbol{\varepsilon}) I$

which in the present case of pure shear where $\operatorname{Tr}(\boldsymbol{\varepsilon})=0$ yields $\sigma_{x x}=111$ $2 G \varepsilon_{x x}$. The dynamic shear modulus is thus $G^{*}=0.5 \sigma_{A} / \varepsilon_{A} \exp (i \delta)$, with the storage and loss moduli given by the real and imaginary parts of $G^{*}: 112$ $G^{\prime}=0.5 \sigma_{A} / \varepsilon_{A} \cos (\delta)$ and $G^{\prime \prime}=0.5 \sigma_{A} / \varepsilon_{A} \sin (\delta)$. We note also that accord- 113 ing to Hooke's law (Eq. (1)), we should have $\sigma_{y y}(t)=\sigma_{z z}(t)=-0.5 \sigma_{x x} 114$ $(t)$, which is readily tested in Fig. 1(b).

115

Fig. 1(c) shows the storage $G^{\prime}$ and loss $G^{\prime \prime}$ shear moduli as a function 116 of temperature. Similar results were obtained by Yu et al. [13]. The stor- 117 age and loss moduli are highly dependent on the loading frequencies, 118 with an $\alpha$-relaxation peak in $G^{\prime \prime}$ close to the glass transition tempera- 119 ture, which shifts to higher temperature as the frequency increases, in 120 agreement with experiments [4,6,34].

121

At variance with previous works, which considered only shear de- 122 formations, we applied four different loading conditions, shown sche- 123 matically in Fig. 2(a):

(1) Constant volume: application of sinusoidal strains $\varepsilon_{x x}(t)=-2 \varepsilon_{y y} 125$ $(t)=-2 \varepsilon_{z z}(t)$ to obtain the shear modulus $G^{*}$, as discussed 126 above.

(2) Uniaxial deformation: application of a sinusoidal strain along the $X 128$ direction and maintain $\sigma_{y y}(t)=\sigma_{z z}(t)=0$ to obtain Young's mod- 129 ulus, $E^{*}$, defined as $\sigma_{x x}(t)=E^{*} \varepsilon_{x x}(t)$.

130

(3) Longitudinal deformation: application of a sinusoidal strain along 131 the $\mathrm{X}$ direction and maintain the $\mathrm{Y}$ and $\mathrm{Z}$ dimensions unchanged 132 to obtain the longitudinal modulus, $M^{*}$, defined as $\sigma_{x x}(t)=133$ $M^{*} \varepsilon_{x x}(t)$.

134

(4) Isostatic deformation: application of the same sinusoidal 135 strain along the $\mathrm{X}, \mathrm{Y}$ and $\mathrm{Z}$ directions simultaneously to obtain 136 the bulk modulus $K^{*}$ defined as $P(t)=-\left(\sigma_{x x}(t)+\sigma_{y y}(t)+137\right.$ $\left.\sigma_{z z}(t)\right) / 3=-3 K^{*} \varepsilon(t)$.

Fig. 2(b) and (c) show the amplitude of the complex moduli $\sigma_{A} / \varepsilon_{A} 140$ and the phase shifts obtained with the different loading conditions. 141 They have opposite hierarchies: a loading condition, which strongly 142 constrains the deformation, such as an isostatic deformation, yields a 143 high $\sigma_{A} / \varepsilon_{A}$ ratio, and a small phase shift and vice versa for a weakly 144 constraining loading, such as uniaxial loading. We also find as expected 145 that above the $\alpha$-relaxation peak, when the system is no longer a glass 146 but is in the supercooled region, Young's and shear moduli drop down 147 to zero and their phase shift increases to almost $\pi / 2$, i.e. the system is 148 now a viscous liquid. But way of contrast, the bulk and longitudinal 149 moduli remain finite at all temperatures, with negligibly small phase 150 shifts.

Hooke's law (Eq. (1)) imposes relations between the elastic mod- 152 uli as listed in Table. 1. For instance, for the longitudinal deformation, 153 $\boldsymbol{\varepsilon}=\left(\begin{array}{lll}\varepsilon & 0 & 0 \\ 0 & 0 & 0 \\ 0 & 0 & 0\end{array}\right)$, such that, from Hooke's law, $\sigma_{x x}=(2 G+\lambda) \varepsilon, \sigma_{y y}$ $=\sigma_{z z}=\lambda \varepsilon$. We therefore have $M=2 G+\lambda$ and $\lambda$ is obtained by 155 monitoring $\sigma_{y y}$ and $\sigma_{z z}$. Fig. 3(a) and (b) show the evolution of $M^{*} 156$ and $\lambda^{*}$ from the NEMD simulations. If the dynamic correspondence 157 
(a)

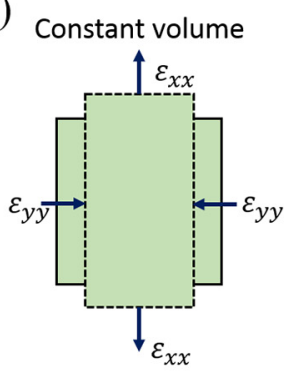

$\varepsilon_{y y}=\varepsilon_{z z}=-\varepsilon_{x x} / 2$

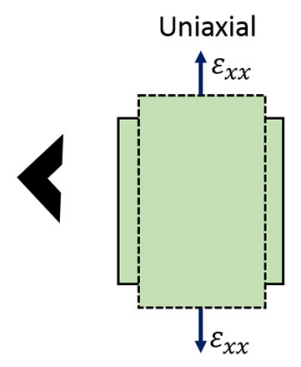

$\sigma_{\mathrm{yy}}=\sigma_{z z}=0$

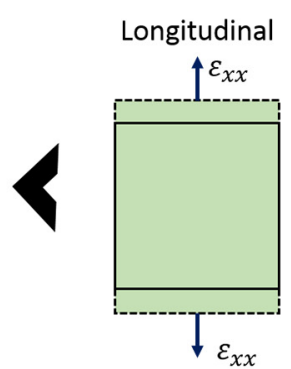

$\varepsilon_{y y}=\varepsilon_{z z}=0$

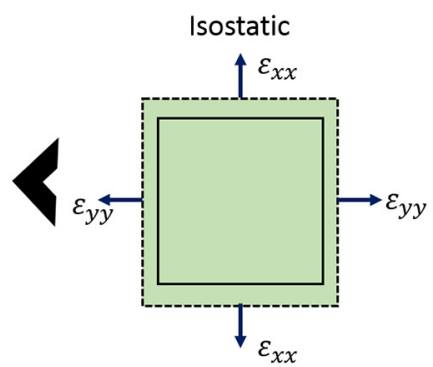

$\varepsilon_{y y}=\varepsilon_{z z}=\varepsilon_{x x}$

Weak

Degree of constraint

Strong
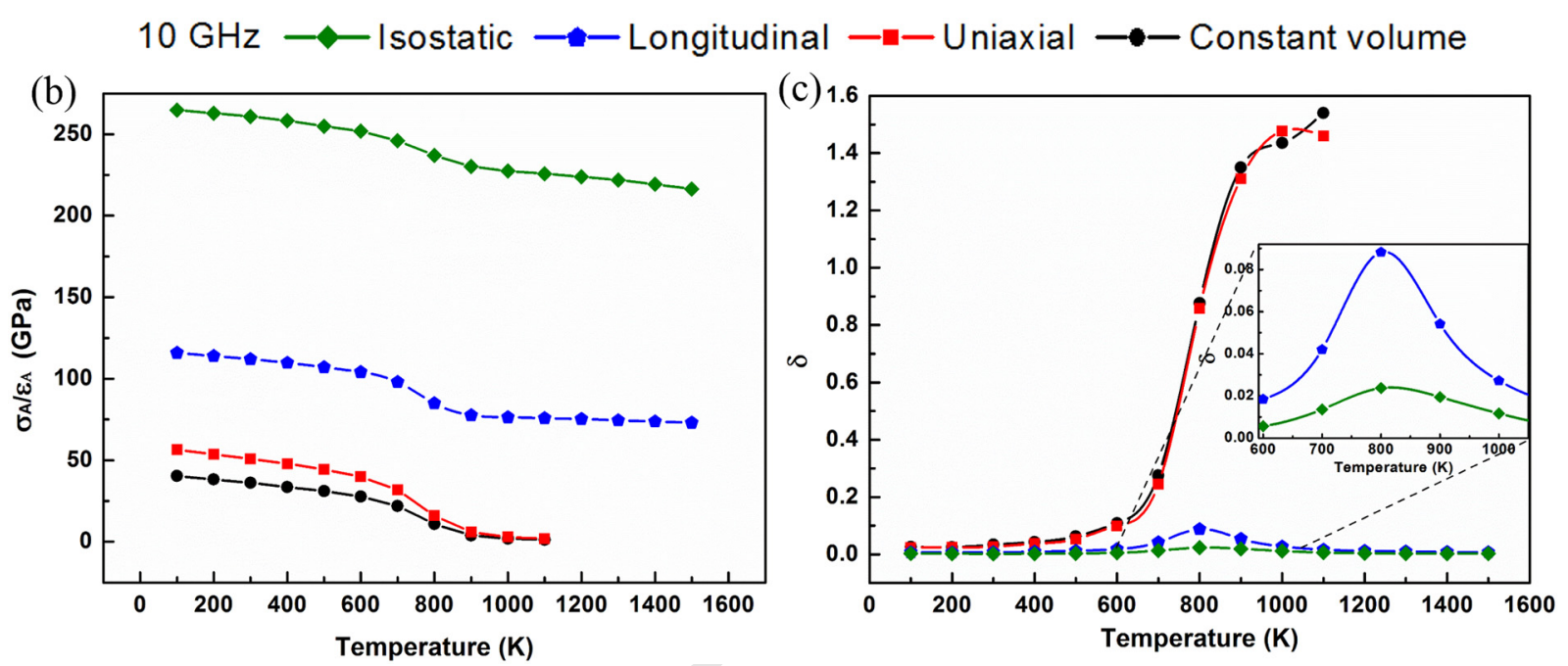

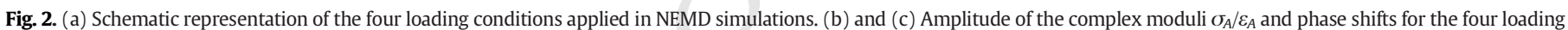
conditions at a frequency of $10 \mathrm{GHz}$.

principle holds, we should have $M^{*}=2 G^{*}+\lambda^{*}$, or equivalently, $G^{*}=$ $\left(M^{*}-\lambda^{*}\right) / 2$, which can be compared to the direct measurement of $G^{*}$ by constant volume deformation. The comparison is done in Fig. 3(c), which shows that the above relation indeed holds at all temperatures. We note that $\lambda^{*}$ is mostly independent of both the temperature and the frequency, with negligible $\lambda^{\prime \prime}$ and phase shift. As a result, there is a constant offset between $M^{\prime}$ and $2 G^{\prime}$ and $M^{\prime}{ }^{\prime} \cong 2 G^{\prime}$. Similarly, in Fig. 3 (d), we verify that $K^{*}=\lambda^{*}+2 G^{*} / 3$. It may not be surprising that these relations satisfy the correspondence principle since they are linear. More complicated is the case of Young's modulus, which is expressed as $E^{*}=$ $G^{*}\left(3 \lambda^{*}+2 G^{*}\right) /\left(\lambda^{*}+G^{*}\right)$. However, Fig. 3(e) shows that this dynamic relation is also verified.

We now turn our attention to the dynamic Poisson's ratio, which has been debated in the literature because it may be defined in different ways [24]. We note that the ratio $\varepsilon_{y y}(t) / \varepsilon_{x x}(t)$ is not sinusoidal and therefore cannot be used to define Poisson's ratio. Instead, we use the ratio of complex strains, $\nu^{*}=-\varepsilon_{y y}{ }^{*} / \varepsilon_{x x}{ }^{*}$. This definition is compared in Fig. 3(f) with two expressions obtained from the correspondence principle: $\nu^{*}=\lambda^{*} / 2\left(\lambda^{*}+G^{*}\right)$ and $\nu^{*}=0.5-E^{*} / 6 K^{*}$. All three

Table 1

Conversion formulas of elastic constants for homogenous isotropic materials.

\begin{tabular}{ccccc}
\hline & $K$ & $E$ & $M$ & $\nu$ \\
\hline$(\lambda, G)$ & $\lambda+\frac{2 G}{3}$ & $\frac{G(3 \lambda+2 G)}{\lambda+G}$ & $\lambda+2 G$ & $\frac{\lambda}{2(\lambda+G)}$ \\
$(K, E)$ & $K$ & $E$ & $\frac{3 K(3 K+E)}{9 K-E}$ & $\frac{3 K-E}{6 K}$
\end{tabular}

expressions lead to the same result, further confirming the reliability 177 of the correspondence principle. We see that $\nu^{\prime}$ reaches 0.5 in the 178 supercooled regime, i.e. the liquid is incompressible. Also, $\nu^{\prime \prime}$ and $\delta_{\nu} 179$ are negative, which implies that the transverse strain lags behind the 180 longitudinal strain under dynamic loading, as expected due to damping 181 effects [35]. A negative $\delta_{\nu}$ is also consistent with $\lambda^{\prime \prime}$ being negligibly 182 small as seen above, since in this case, $\tan \delta_{\nu} \simeq-G^{\prime \prime} /\left(\lambda^{\prime}+G^{\prime}\right)$. Negative 183 $\delta_{\nu}$ were observed in previous experimental works, directly measuring 184 the dynamic Poisson's ratio of several polymers $[23,35]$. Since the 185 phase shift is very small, an extreme accuracy of the measurements is 186 needed and some works have also reported zero phase shift [36,37]. 187

Atomic mobility is the key to understand structural relaxation in 188 MGs [13]. Fig. 4(a) shows an example of non-affine atomic 189 displacements between the end of the 1 st and the 5 th cycles, when 190 the cell is back to its original shape and there is no displacement in- 191 duced by the applied homogeneous deformation. Atoms in different re- 192 gions have very different mobilities, as expected from dynamic 193 heterogeneities [38]. Following the work of Yu et al. [13], we define 194 "faster atoms" as having displacements larger than $1.4 \AA$ A, half the aver- 195 age nearest neighbor distance. The "slow atoms" shown in Fig. 4196 (b) have almost reversible displacements under the deformation, and 197 therefore contribute only to the elastic deformation of the MG. Con- 198 versely, the "faster atoms" undergo irreversible movements during the 199 loading, i.e. inelastic deformation, which leads to energy dissipation. 200 To further reveal the structural origin of the dynamic heterogeneity, 201 Fig. 4(c) shows the $\mathrm{Cu}$ atoms with Voronoi index $\langle 0,0,12,0\rangle$, identified 202 as the central atoms of full icosahedral clusters. By deleting the isolated 203 atoms with a cutoff $3.7 \AA$ (the first minimum of the radial distribution 204 

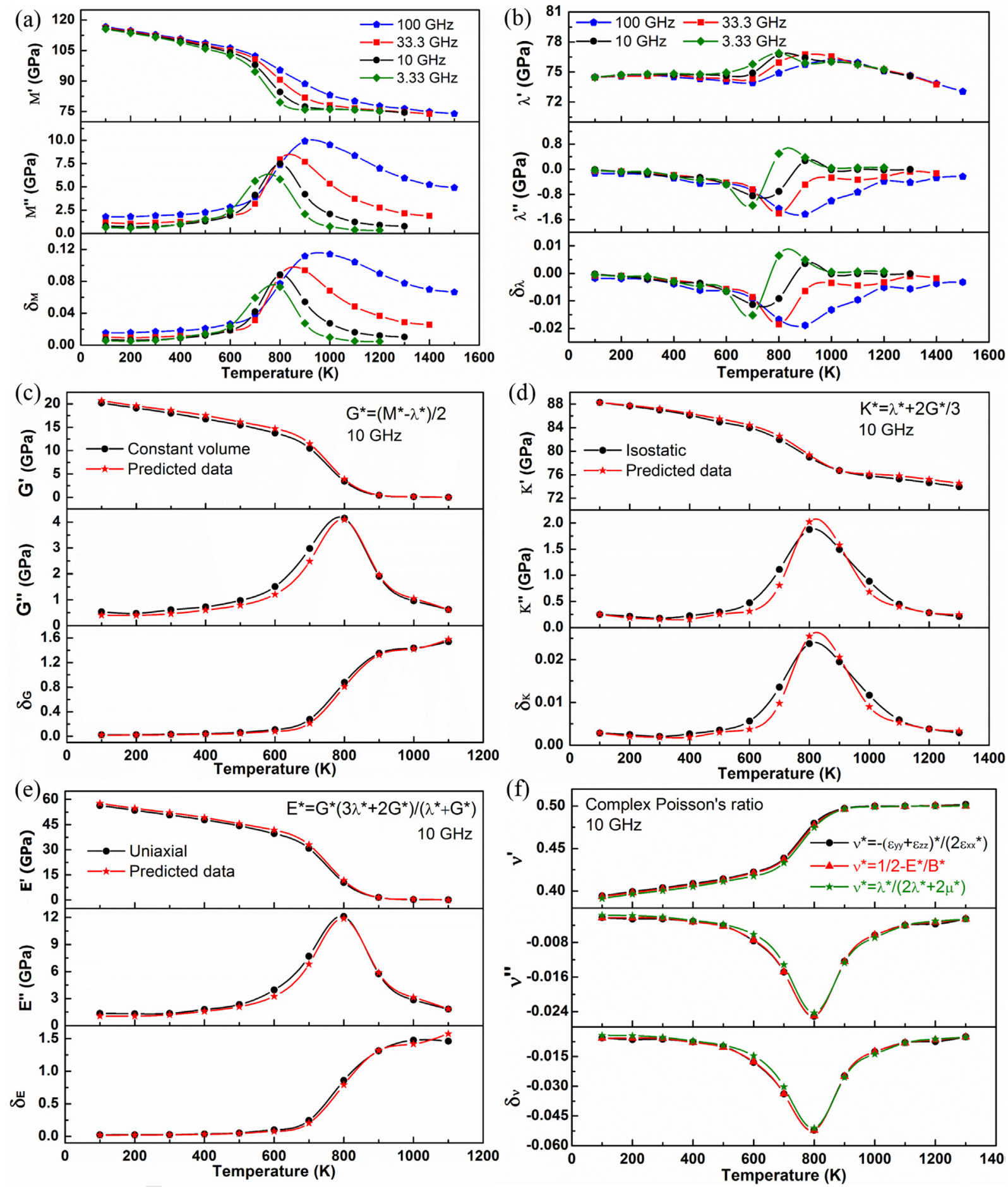

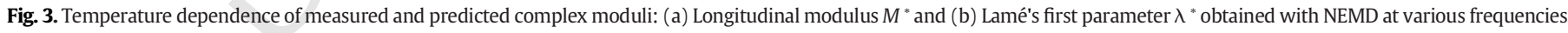

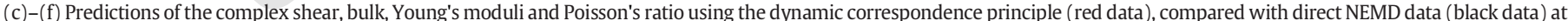
$10 \mathrm{GHz}$. (For interpretation of the references to color in this figure legend, the reader is referred to the web version of this article.)

function), not only the "slow atoms" on the surface of the simulation box but also the atoms with reversible movements inside the box match very well, as shown comparing Fig. 4(b) and (d). This result is consistent with the work of Yu et al. [13]. It can be concluded that the Cu-centered full icosahedral clusters in CuZr MG are stable and have a low atomic mobility, contributing to the stored elastic energy in each loading cycle. Conversely, most other atoms have larger mobilities and undergo irreversible inelastic displacements, contributing to the viscoelastic component and the energy dissipated in each loading cycle.
In summary, our results provide a numerical verification of the dy- 214 namic correspondence principle by investigating $\mathrm{Cu}_{64} \mathrm{Zr}_{36}$ MG in 215 NEMD simulations. The resulting relationships between the viscoelastic 216 complex moduli provide a guidance for engineering applications of vis- 217 coelastic materials under multi-axial stress conditions. Moreover, a 218 well-posed definition of dynamic Poisson's ratio is highlighted. Spa- 219 tially, the Cu-centered icosahedral clusters are found stable under cyclic 220 deformation, contributing to the elastic component of the deformation, 221 while most other atoms undergo irreversible displacements and 222 

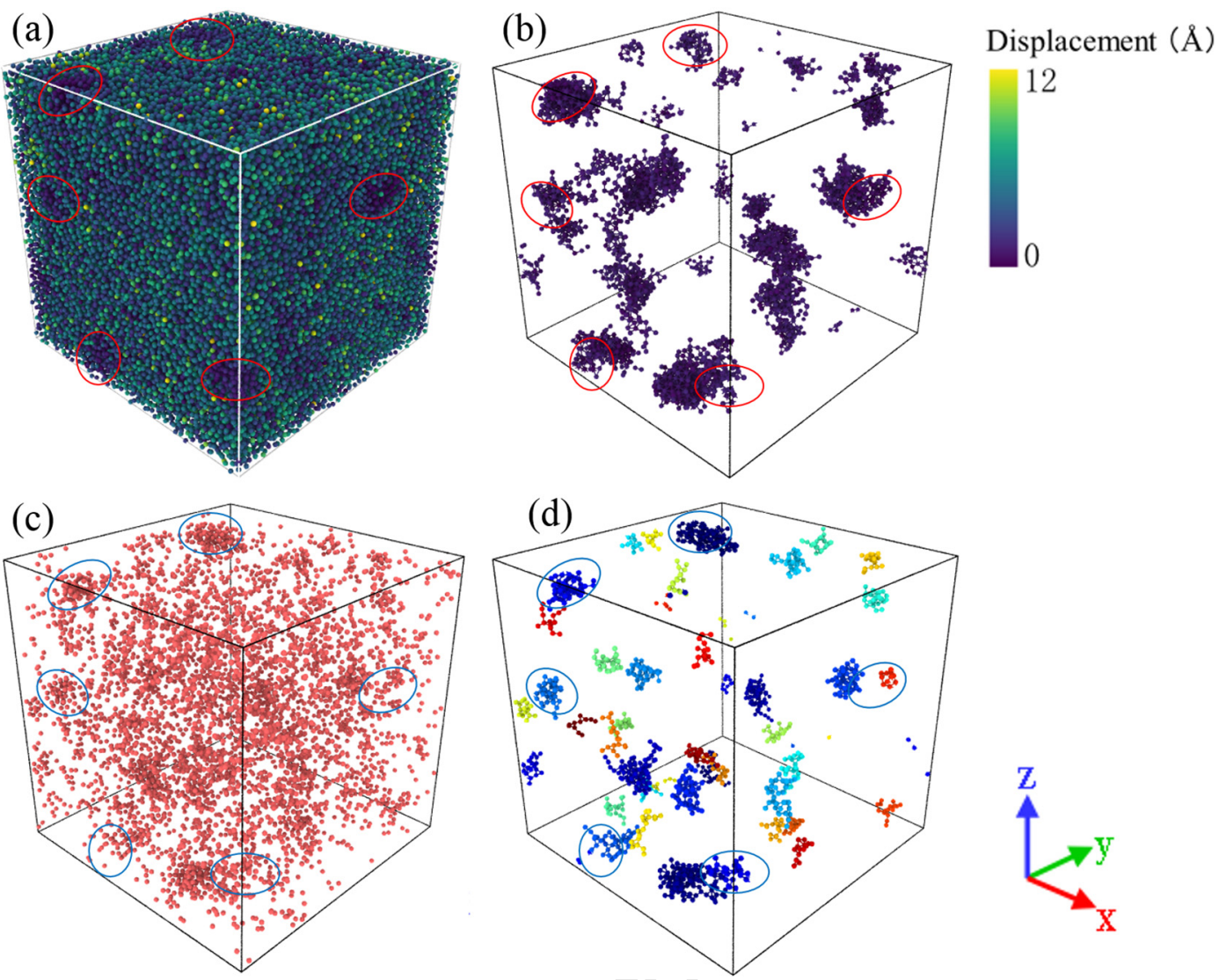

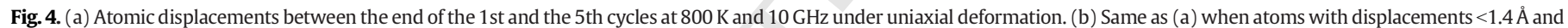

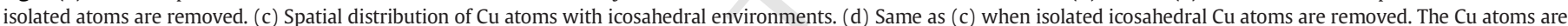

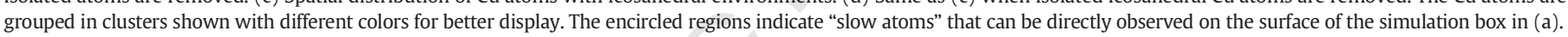

contribute to the viscoelastic part. Our findings make a contribution to better understand the dynamic mechanical relaxations of MGs and also convey important information on the microstructural processes occurring during the glass transition.

\section{Acknowledgements}

This work was performed using HPC resources from the FLMSN, "Fédération Lyonnaise de Modélisation et Sciences Numériques", partner of EQUIPEX EQUIP@MESO. GJL acknowledges China Scholarship Council (CSC) for the scholarship. The research of JCQ was supported by the Fundamental Research Funds for the Central Universities (Nos. 3102018ZY010 and 3102017JC01003). YY acknowledges the Alexander von Humboldt Foundation for supporting his stay at Max-PlanckInstitut für Eisenforschung.

\section{Appendix A. Supplementary data}

Supplementary data to this article can be found online at https://doi. org/10.1016/j.scriptamat.2019.08.015.

\section{References}

[1] K.P. Menard, Dynamic Mechanical Analysis: A Practical Introduction, CRC press, Boca Raton, Florida, 2008

[2] M.W. Chen, NPG Asia Mater. 3 (2011) 82-90.

[3] X.Q. Zhou, D.Y. Yu, X.Y. Shao, S.Q. Zhang, S. Wang, Compos. Struct. 136 (2016) 460-480.

[4] J.C. Qiao, J.M. Pelletier, J. Mater. Sci. Technol. 30 (2014) 523-545.

[5] J.C. Qiao, Q. Wang, J.M. Pelletier, H. Kato, R. Casalini, D. Crespo, E. Pineda, Y. Yao, Y. Yang, Prog. Mater. Sci. 104 (2019) 250-329.

[6] H.B. Yu, W.H. Wang, K. Samwer, Mater. Today 16 (2013) 183-191.
[7] G.J. Lyu, J.C. Qiao, J. Gu, M. Song, J.-M. Pelletier, Y. Yao, J. Alloy. Compd. 769 (2018) 249 $443-452$.

[8] M. Lee, Y. Li, Y. Feng, C. Carter, Intermetallics 10 (2002) 1061-1064.

[9] X. Liu, B. Zhang, P. Wen, W. Wang, J. Non-Cryst. Solids 352 (2006) 4013-4016.

[10] G.J. Lyu, J.C. Qiao, J.M. Pelletier, Y. Yao, Mater. Sci. Eng. A 711 (2018) 356-363.

[11] J.C. Qiao, J.M. Pelletier, J. Appl. Phys. 112 (2012), 033518.

[12] J.C. Qiao, Y. Yao, J.M. Pelletier, L.M. Keer, Int. J. Plast. 82 (2016) 62-75.

[13] H.-B. Yu, K. Samwer, Phys. Rev. B 90 (2014), 144201.

[14] B.S. Shang, J. Rottler, P.F. Guan, J.-L. Barrat, Phys. Rev. Lett. 122 (2019), 105501.

[15] N.V. Priezjev, Comput. Mater. Sci. 153 (2018) 235-240.

[16] R. Ranganathan, Y. Shi, P. Keblinski, Phys. Rev. B 95 (2017), 214112.

[17] Z.D. Sha, L.C. He, O.X. Pei, Z.S. Liu, Y.W. Zhang, T.J. Wang, Scr. Mater 83 (2014) 37-40, 260

[18] L.D. Landau, E. Lifshiëtìs, A. Kosevich, L. Pitaevskiæi, Theory of Elasticity, Pergamon 261 Press, Oxford, 1986.

[19] W.T. Read, J. Appl. Phys. 21 (1950) 671-674 263

[20] N.W. Tschoegl, The Phenomenological Theory of Linear Viscoelastic Behavior: An In- 264 troduction, Springer Science \& Business Media, Berlin Heidelberg, 2012.

[21] N.W. Tschoegl, W.G. Knauss, I. Emri, Mech. Time-Depend. Mat. 6 (2002) 3-51. 266

[22] R.S. Lakes, A. Wineman, J. Elast. 85 (2006) 45-63.

[23] M. Giovagnoni, Mech. Mater. 17 (1994) 33-46.

[24] H.H. Hilton, Mater. Sci. Appl. 8 (2017) 291

24] T. Damart, A. Tanguy, D. Rodney, Phys. Rev. B 95 (2017), 054203.

[27] D. Wang, Y. Li, B.B. Sun, M.L. Sui, K. Lu, E. Ma, Appl. Phys. Lett. 84 (2004) 4029-4031. 272

[28] D. Xu, B. Lohwongwatana, G. Duan, W.L. Johnson, C. Garland, Acta Mater. 52 (2004) 273 2621-2624.

[29] S.D. Feng, L. Qi, L.M. Wang, S.P. Pan, M.Z. Ma, X.Y. Zhang, G. Li, R.P. Liu, Acta Mater. 95275 (2015) 236-243.

[30] S.D. Feng, L. Qi, L.M. Wang, P.F. Yu, S.L. Zhang, M.Z. Ma, X.Y. Zhang, Q. Jing, K.L. Ngai, 277 A.L. Greer, G. Li, R.P. Liu, Scr. Mater. 115 (2016) 57-61.

[31] T. Brink, M. Peterlechner, H. Roesner, K. Albe, G. Wilde, Phys. Rev. Appl. 5 (2016), 279 054005.

[32] Y.Q. Cheng, E. Ma, Prog. Mater. Sci. 56 (2011) 379-473.

[33] V. Borovikov, M.I. Mendelev, A.H. King, Model. Simul. Mater. Sci. Eng. 24 (2016), 282 085017.

[34] J.C. Oiao, J. Cong Q Wang J.M. Pelletier, Y Yao, J. Alloy. Compd. 749 (2018) 262-267. 284

[35] T. Pritz, Appl. Acoust. 60 (2000) 279-292.

[36] P.S. Dubbelday, J. Acoust. Soc. Am. 91 (1992) 1737-1744.

[37] G. Ledon, J. Derouet, J. Sound Vib. 89 (1983) 155-167.

[38] L. Berthier, Physics 4 (2011) 42. 